\title{
A NEW OPTICAL METHOD FOR STUDYING FLAMES
}

\author{
R. BERNARD and D. MAS \\ Université Claude-Bernard, Lyon I, 69621 Villeurbanne, France
}

(Reçu le 18 septembre 1972)

\begin{abstract}
Résumé. - On utilise le filtrage des fréquences spatiales pour mettre en évidence les turbulences
\end{abstract} moyennes dans une flamme.

Abstract. - A spatial-filtering set-up is used in order to visualize mean turbulences in a flame.

Flames are not easy to photograph. But, by their temperature and concentration gradients they may be considered as phase objects and are subject to several optical treatments [1].

In one of them, a source of white light illuminates a diffusing screen in front of which is placed a photograph of rectilinear fringes. The camera is focused on the fringes visible through the phase object as a succession of irregular lines.

In a second operation, a beam of monochromatic light is made to converge on the photograph previously obtained which can be considered as a collection of elementary gratings with different spacing values. The gratings with spacing not too different from the spatial period of the unmodulated fringes help to illuminate a slit lined up on the first order diffracted spectrum.

The zones showing alteration corresponding to contours of the object do not diffract any light in this direction and look black on a clear background [2]. If we use white light for this reconstruction, the studied phase object appears with a coloured contrast.

In a previous work on flames [3], the photograph was recorded during a $1 / 1000 \mathrm{~s}$ flash. In this letter, on the other hand, we show the possibility of obtaining other results by temporal integration of the optical path fluctuations associated with temperature and pressure.

The flame of an ordinary gas burner has been photographed in front of a very fine metallic wire gauze. But in this case, the photographic emulsion has been replaced by a photopolymer and the exposure is very long. The resulting plate, after proper treatment, may be considered as a bidimensional diffraction grating. However the parts of the gauze which were situated behind the more perturbated regions of the flame, have not been recorded since their successive images have been blurred by the random fluctuations of the optical medium during the exposure. Accordingly, they appear dark in the beams diffracted by the plate. For example, the figure shows a photograph of the plate obtained with one of the horizontal

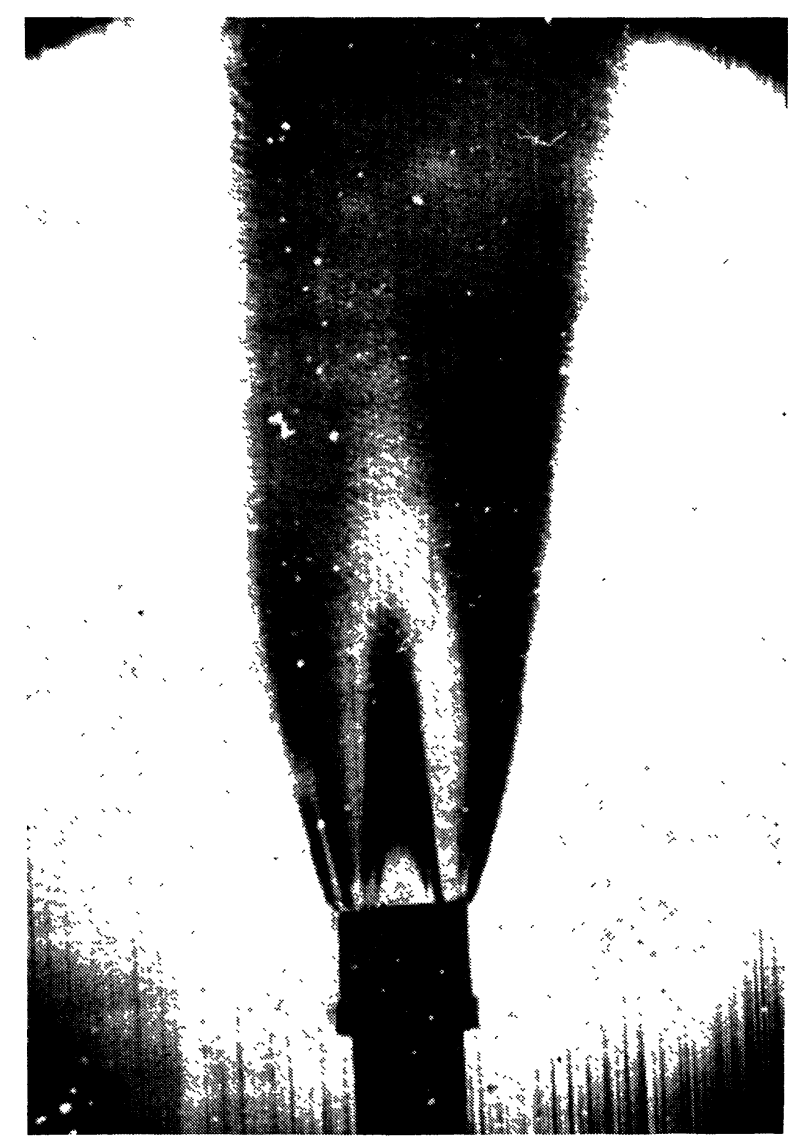

FIG. 1. 
first-order spectra. Its dark zones correspond to the mean maximum turbulences in the flame.

Here are some technical details relative to this experiment :

Spatial period of the gauze : $50 \mu$.

Wire width of the gauze : $25 \mu$.
Lighting: $1000 \mathrm{~W}$ high-pressure mercury lamp behind a diffuser.

Photopolymer : Kodak KAR 3 autopositive undiluted solution.

Camera : $\mathrm{f} / 4$ aperture, $1.3 \mathrm{~m}$ from the flame. Exposure : $70 \mathrm{mn}$.

\section{References}

[1] Weinberg, F. J., Optics of Flames (Butterworths London) 1963.

[2] Bernard, R. and Gacon, J. C., Nouv. Revue Opt. Appl. 2 (1971) 51.

[3] Bernard, R. and GaCon, J. C., Nature 226 (1970) 447. 